\title{
Adult Respiratory Distress Syndrome and alveolar epithelium apoptosis: an histopathological and immunohistochemical study
}

\author{
Š. Tóth1, S. Pingorová2 ${ }^{2}$ Z. Jonecová1, R. Morochovič ${ }^{2}$, M. Pomfy¹, J. Veselá1 \\ 1Department of Histology and Embryology, Faculty of Medicine, Šrobárova 2, \\ Pavol Jozef Šafárik University, Košice, Slovak Republic, \\ ${ }^{2}$ Department of Traumatology, Rastislavova 43, Pavol Jozef Šafárik University, Košice, Slovak Republic
}

\begin{abstract}
Adult Respiratory Distress Syndrome results from a variety of different initial insults, including trauma, sepsis, pneumonia and aspiration, and represents a severe form of acute lung injury. The lung samples of a 20-year-old man who had suffered a serious motorbike accident were obtained for histological examination. He died on the seventh day as a consequence of respiratory failure. The typical histopathological features of syndrome overlapping the first exudative phase into the second proliferate phase were observed. The apoptotic index of the early apoptotic phase evaluated using M30CytoDEATH was 3.4 \pm 0.2 . The average number of apoptotic cells in the intermediate and late phases measured using the TUNEL method was 9.8 \pm 0.7. Our findings indicate that alveolar epithelium apoptosis seems to be less important during the early phases of Adult Respiratory Distress Syndrome.
\end{abstract}

Key words: ARDS, pneumocyte, apoptosis, TUNEL, M30CytoDEATH

\section{Introduction}

Adult Respiratory Distress Syndrome (ARDS) is characterized by diffuse pulmonary microvascular injury resulting in increased permeability and thus also in non-cardiogenic pulmonary oedema. This clinical syndrome determines the host inflammatory and repair cellular responses, but the mechanisms regulating the resolution of ARDS are however poorly understood. The mortality rate remains high, ranging between $20 \%$ and $60 \%$ [1]. The basic abnormality is the disruption of the normal alveolar-capillary barrier. The triggering insult in the parenchyma is considered to be an initial release of proinflammatory cytokines and other inflammatory mediators, secreted by local epithelial and endothelial cells. Activated neutrophils, macrophages, platelets and lymphocytes quickly migrate into the inflamed lung parenchyma and contribute to the amplification of the phenomenon. The histopathological picture involves features of diffuse alveolar damage and hyaline membrane formation.

Correspondence: $\breve{S}$. Tóth, Department of Histology and Embryology, Faculty of Medicine, Šrobárova 2, Pavol Jozef Šafárik University, 04180 Košice, Slovak Republic; tel.: (+420 37) 0556222606, e-mail: s_toth2000@yahoo.com
The histopathological pattern can be divided into 3 main phases: the first early exudative, the second proliferative phase and the last fibrotic phase $[2,3]$. These phases can overlap and diffuse into each other without apparent borders. The purpose of this immunohistochemical study was to evaluate the histopathological alterations and to determine the apoptosis of the alveolar epithelium using immunohistochemical methods.

\section{Materials and methods}

Clinical features. A motorcycle rider (20-year-old male) was involved in a high-energy collision with a car, suffering traumatic amputation of the upper right arm in its middle third, compound fractures of the right thigh and right tibia with soft tissue devastation and problematic blood perfusion in the region. Patient was in haemorrhagic shock with $40 \%$ estimated blood loss (initial haemoglobin $59 \mathrm{~g} / \mathrm{l}), 80 / 40 \mathrm{mmHg}$ blood pressure, 120 beats per minute heart rate and mental confusion. Spiral head and torso CT scan revealed no other organ injury. After surgical soft tissue debridement and bone stabilization (external fixator, Kirschner-wire skeletal traction) the patient was transferred to the intensive care unit for

Abbreviations: ARDS - Adult Respiratory Distress Syndrome; PEEP - positive end-expiratory pressure; $\mathrm{H}+\mathrm{E}$ - hematoxylin \& eosin 
further treatment. On the second day no signs of lung parenchyma infiltration were revealed by plain X-ray of the chest. There was an unsuccessful attempt to wean the patient from mechanical ventilation. On the third day after injury, diffuse crackles on chest auscultation and infiltration of the lung parenchyma at the right and left bases with right pleural effusion were revealed by X-ray. Positive end-expiratory pressure (PEEP) mode of mechanical ventilation was instigated. On the fourth day the patient became cyanotic with a drop in oxygen saturation to $72 \%$. Emergency therapeutic fibreoptic bronchoscopy was carried out with suctioning of pink-tinged clear frothy fluid, and the right pleural space was drained with active suction. On the fifth day a CT scan of the lungs revealed signs of ARDS, atelectasis and minimal bilateral pleural effusions. Despite treatment with higher levels of PEEP with an increased fraction of inspired oxygen in the gas and repeated therapeutic fibreoptic bronchoscopies, the patient died from his injuries on day seven.

Specimen samples. Necroptic lung specimens were harvested and immediately fixated in $4 \%$ paraformaldehyde and embedded in Paraplast wax, sectioned in $4-5 \mu \mathrm{m}$ tissue slices and stained with $\mathrm{H}+\mathrm{E}$. The severity of lung injury was determined. Two selective immunohistochemical methods (both Roche Molecular Biochemicals, Germany) were used - M30CytoDEATH and terminal deoxyuridine In Situ nick and end-labeling method (TUNEL) for apoptotic cell measurement.

A lung histopathological injury scoring method was applied to quantify changes in lung architecture visible by light microscopy. The degree of microscopic injury was scored based on the following variables: alveolar and interstitial oedema, leukocytes infiltration, alveolar haemorrhage, presence of fibrin and septal thickening. The severity of injury was graded for each variable: no injury $=0$; injury to $25 \%$ of the field $=1$; injury to $50 \%$ of the field $=2$; injury to $75 \%$ of the field $=3$; and diffuse injury to $>75 \%$ of the field $=4[4]$. The samples were analyzed based on a scaled grading system by a pathologist who was blinded to the pathological state and the region of sampling.

\section{Results}

\section{Histopathological and immunohistochemical findings}

In the lung $\mathrm{H}+\mathrm{E}$ stained sections a high degree of fourth-grade histological injury- severe diffuse injury up to $>75 \%$ of the field - was displayed (Fig. 1). Accentuated interstitial and intraalveolar oedema, destruction of the alveolar epithelium with denudation of the basement membrane, capillary congestion and multiplication of alveolar macrophages, interstitial inflammation and many focal intraalveolar haemorrhages were observed. The walls of the alveoli were necrotic and occasionally lined with amorphous eosinophilic material, probably consisting largely of fibrin and formed the hyaline membranes. The capillaries were full of blood, but endothelial and alveolar epithelial cells could not be distinguished. The alveolar spaces contained necrotic cells and cell debris. Type II pneumocytes multiplied to line the alveolar surfaces, becoming cuboidal in shape. Mixed inflammatory infiltration was present in the interstitium. A lot of macrophages were very large and distended with hemosiderin derived from the ingested red blood cells.
There were also early organization and fibrosis with elongated fibroblasts in the alveolar walls and within the alveoli (Fig. 2). Our histopathological observation corresponds with typical features of the first acute exudative as well as the second proliferative ARDS phases.

The average numbers of positive apoptotic alveolar epithelial cells in the lung parenchyma were detected using the M30 method, and only a low number 3.4 \pm 0.2 (Fig. 3) was found. In contrast, a high number $9.8 \pm 0.7$ (Fig. 4) was detected using the TUNEL method. All apoptotic positive cells were dispersed in the lung parenchyma. Alveolar macrophages with M30-positive cytoplasm (resulting from phagocytosis of apoptotic cells or apoptotic bodies), and granulocytes with TUNEL-positive segmented nuclei, were occasionally present.

\section{Discussion}

Data published within the last ten years have revealed the complexity of lung cell apoptosis control mechanisms. Although apoptosis plays an important role in the immune system and during tissue repair, there are several potential mechanisms by which apoptosis might play a role in ARDS. Apoptosis was described during ARDS particularly in the population of neutrophils [5], endothelial cells [6], myofibroblasts [7], and pericytes [8], as well as in alveolar epithelial cells [9].

The apoptotic alveolar epithelial cells in the presented case showed the maximum positive immunoreactivity detected using the TUNEL method $(9,8 \pm 0,7)$. The immunureactivity detected with the M30 method was markedly lower $(3,4 \pm 0,2)$, although the statistical significance was not confirmed. The difference was more likely caused by different detection sensitivities of the immunohistochemical methods used. The M30 method shows a neo-epitope in CK18 typical for an early caspase cleavage event [10], which foresees DNA fragmentation in the nucleus, evaluated with the TUNEL method. In our previous study [11], we determined the apoptotic index in the lungs of healthy rats. During the early phase we measured an apoptotic index of $0.75 \pm 0.14$ and of $0.4 \pm 0.16$ in the late phase. Apoptosis represents one of the important mechanisms maintaining homeostasis in live organisms, and normally occurs throughout life. No significant correlation between apoptosis and clinical physiological function of the lungs has been confirmed. Fisher et al. [9] found that up to $34 \%$ of pulmonary cells underwent apoptotic cell death after transplantation of lungs without significant alteration of their function in humans. The authors hypothesized that a high index of apoptosis in transplanted organs indicates their ability to eliminate injured cells and avoid the more destructive process of necrosis. In the presented case study, the 

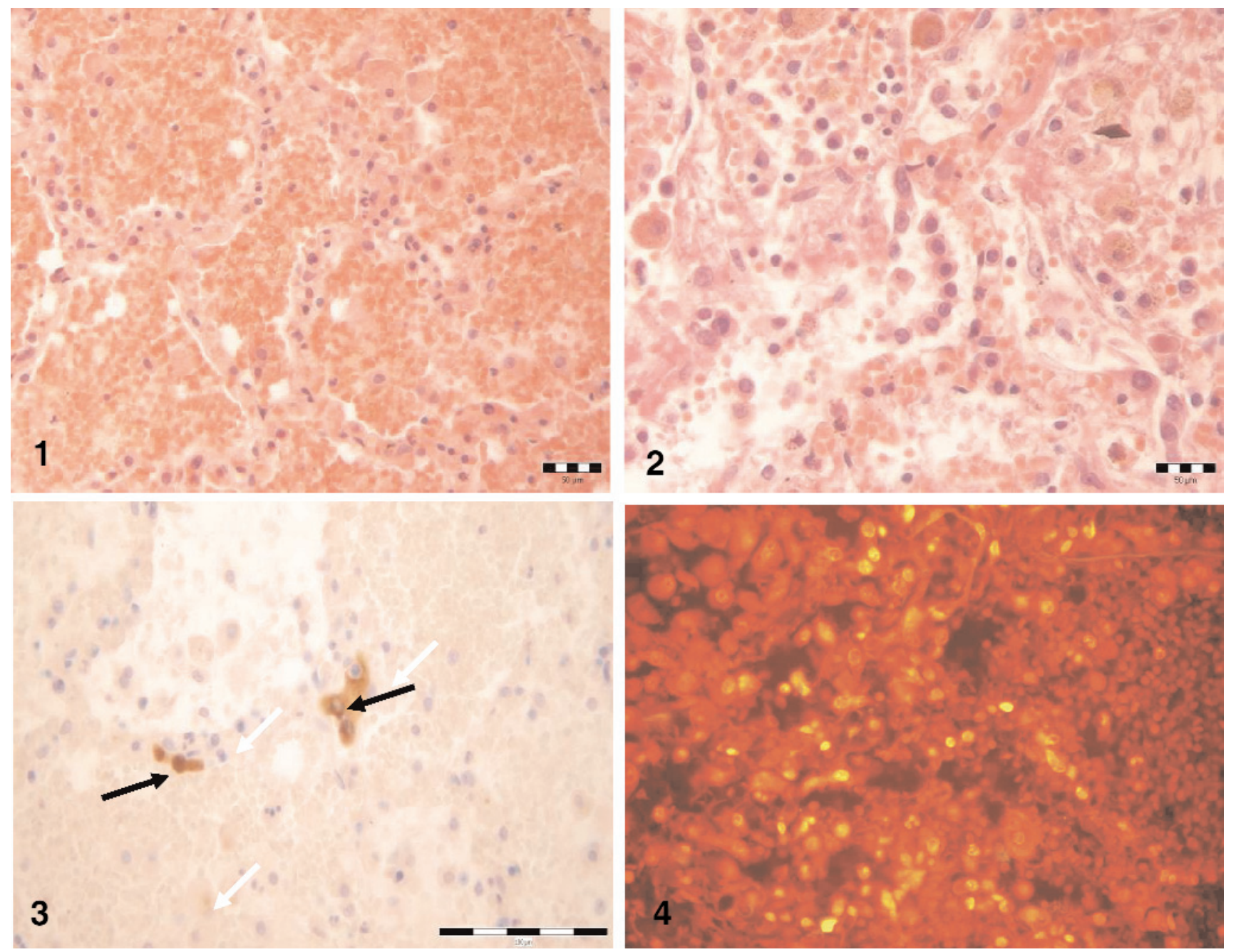

Fig. 1. The alveolar spaces were filled with densely-packed red blood cells and the alveolar septa showed necrotic signs. The capillaries were full of blood, but the endothelial and alveolar epithelial cells could not be clearly distinguished $(\mathrm{H}+\mathrm{E}$, magnification $\times 400)$.

Fig. 2. The capillaries were full of blood cells. The alveolar spaces contained necrotic cells and cell debris, much of it derived from red blood cells. The airspaces showed incipient organization of the hyaline membranes by proliferation of fibroblasts and acute and chronic inflammatory cells. The newly-formed type II pneumocytes tended to have a prominent cuboidal shape and they formed rows. There were abundant fibrin fibres present. The alveolar lining cells and capillary endothelial cells were sloughed, leaving their respective basement membranes denuded of cells $(\mathrm{H}+\mathrm{E}$, magnification $\times 400)$.

Fig. 3. M30 immunohistochemical staining showed only a low number of apoptotic cells. M30-positive cells (arrows) were detected by brown-coloured cytoplasm based on reaction of the M30 antibody with altered cytoskeleton during the early apoptosis stage (M30 CytoDEATH, cytokeratin 18, magnification $\times 400$ ).

Fig. 4. Representative view of lung necropsy stained using the In Situ TUNEL method. The bright-yellow apoptotic cells (arrows) were found in the alveolar epithelium as well as within the interstitium (In Situ TUNEL staining method; magnification $\times 400$ )

major form of cell death appeared to be necrosis visualized using the $\mathrm{H}+\mathrm{E}$ staining method. The histopathological finding revealed diffuse injury to $>75 \%$ of the field. Our results indicate that the cleavage and disruption of CK18 in the alveolar epithelium is of lesser importance in acute lung injury because only low numbers of M30-positive apoptotic alveolar epithelial cells were detected.

Two main hypotheses involving apoptosis in the pathogenesis of ARDS have been formulated: the 'neutrophilic hypothesis' and 'epithelial hypothesis'. The epithelial hypothesis suggests association of epithelial injury during ARDS with apoptotic death of alveolar epithelial cells in response to soluble mediators such as soluble Fas-ligand. The blocking of such inhibitors may be beneficial in preventing or treating ARDS [12]. The neutrophilic hypothesis suggests an important role of neutrophil apoptosis in the resolution of inflammation [13]. The precise mechanism responsible for epithelial cell apoptosis in acute lung injury has not been understood so far, but several lines of evidence point to the role of the Fas/Fas ligand system [14].

According to Polunovsky et al. [15], apoptosis may play an important role during resolution of lung injury 
mainly in the late fibrotic phase of ARDS. Endothelial apoptosis may play a role in the pathogenesis of ARDS, and its inhibition may provide clinical benefits for patients with ARDS [6]. Our data indicate that during the overlapping exudative and proliferative stages of ARDS, the relative rate of apoptotic cell death should be lower than the rate of other forms of cell death.

In conclusion, the histological picture of ARDS was a predominant characteristic of the presented study. The detected apoptosis of alveolar epithelial cells may contribute to the pathogenesis of ARDS. The higher apoptotic index in the alveolar epithelium was found using the TUNEL staining method, which is sensitive and specific for intermediate and late stages of apoptosis in cell nuclei. Our findings indicate that apoptotic cell death is important at the beginning of the second proliferative phase of ARDS, but that serious destruction of the alveolar epithelium plays a central role.

Acknowledgements: We gratefully acknowledge the material and technical assistance of A. Hantke, L'. Háberová and A. Horňáková. This work was supported by VEGA grant 1/4230/07 and APVV grant 0252-07.

\section{References}

[1] Rubenfeld G, Caldwell E, Peabody E, Weaver J, Martin D, Neff M. Incidence and outcomes of acute lung injury. $N$ Engl J Med. 2005;353:1685-1693.

[2] Cagle P, Allen T, Barrios R, Bedrossian C, Haque A, Laga A. Color atlas and text of pulmonary pathology. Philadelphia: Lippincott Williams \& Wilkins; 2005.

[ 3] Wright JL. The pathology of ARDS. In: Russell JA, Walley KR eds. Acute respiratory distress syndrome. A comprehensive clinical approaach. New York: Cambridge University Press; 1999:48-62.

[4] Su X, Bai C, Hong Q, Zhu D, He L, Wu J, et al. Effect of continuous hemofiltration on hemodynamics, lung inflammation and pulmonary edema in a canine model of acute lung injury. Intensive Care Med. 2003;29:2034-42.
[5] Fialkow L, Fochesatto Filho L, Bozzetti M, Milani AR, Rodrigues Filho E, Ladniuk R. Neutrophil apoptosis: a marker of disease severity in sepsis and sepsis-induced acute respiratory distress syndrome. Crit Care. 2006;10:155.

[ 6] Abadie Y, Bregeon F, Papazian L, Lange F, Chailley-Heu B, Thomas P. Decreased VEGF concentration in lung tissue and vascular injury during ARDS. Eur Respir J. 2005;25:139-46.

[ 7] Ware L, Matthay M. The acute respiratory distress syndrome. N Engl J Med. 2000;342:1334-1349.

[ 8] Edelman D, Jiang Y, Tyburski J, Wilson R, Steffes C. Pericytes and their role in microvasculature homeostasis. J Surg Res. 2006;135:305-11.

[9] Fisher S, Cassivi SD, Xavier AM, Cardella JA, Cutz E, Edwards V. Cell death in human lung transplantation: Apoptosis induction in human lungs during ischemia and after transplantation. Annals of Surgery. 2000;231:424-431.

[10] Macfarlane M, Merrison W, Dinsdale D, Cohen G. Active caspases and cleaved cytokeratins are sequestered into cytoplasmic inclusions in TRAIL-induced apoptosis. J Cell Biol. 2000;148:1239-54.

[11] Tóth Š, Pomfy M, Veselá J, Wohlfahrt P, Pingorová S, Beňačka R. Cell death in lung parenchyma after intestinal ischemia reperfusion. In: Programme and Abstract Book of the 43rd International Congress on Anatomy and 43rd Lojda Symposium on Histochemistry: Morphology 2006, Praque, September 3-6, 2006:166.

[12] Matute-Bello G, Liles W, Steinberg K, Kiener P, Mongovin S, Chi E. Soluble Fas-ligand induced epithelial cell apoptosis in humans with acute lung injury (ARDS). $J$ Immunol. 1999; 163:2217-2225.

[13] Haslet C, Savill J, Whyte M, Stern M, Dransfield I, Meagher L. Granulocyte apoptosis and the control of inflammation. Philos Trans R Soc Lond B Biol Sci. 1994;345:327-333.

[14] Matute-Bello G, Liles W, Frevert C, Nakamura M, Ballman $\mathrm{K}$, Vathanaprida C. Recombinant human Fas-ligand induces alveolar epithelial cell apoptosis and lung injury in rabbits. Am J Physiol. 2001;281:328-335.

[15] Polunovsky V, Chen B, Henke C, Snover D, Wendt C, Ingbar D. Role of mesenchymal cell death in lung remodeling after injury. J Clin Invest. 1993;92:388-397.

Submitted: 31 March, 2009 Accepted after review: 17 July, 2009 